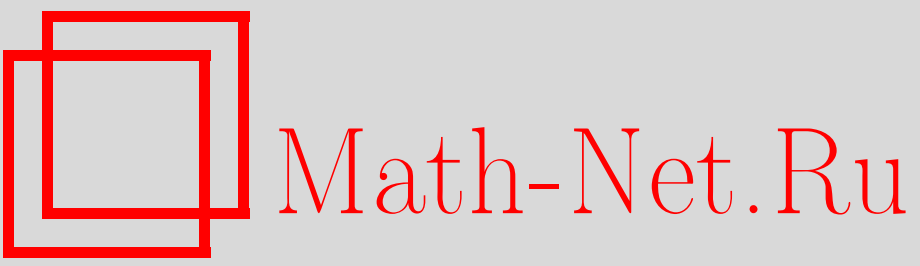

А. В. Латышев, А. А. Юшканов, Кинетическое уравнение для квантовых фермигазов и аналитическое решение граничных задач, ТМФ, 2003, том 134, номер 2, 310-324

DOI: https://doi.org/10.4213/tmf152

Использование Общероссийского математического портала Math-Net.Ru подразумевает, что вы прочитали и согласны с пользовательским соглашением

http://www.mathnet.ru/rus/agreement

Параметры загрузки:

IP: 18.209 .158 .208

26 апреля 2023 г., 17:15:16 


\author{
ТЕОРЕТИЧЕСКАЯ \\ И МАТЕМАТИЧЕСКАЯ \\ ФИЗИКА \\ Том 134, № 2 \\ февраль, 2003
}

(C) 2003 г. $\quad$ А.В. Латышев* , А. А. Юшканов*

\title{
КИНЕТИЧЕСКОЕ УРАВНЕНИЕ ДЛЯ КВАНТОВЫХ ФЕРМИ-ГАЗОВ И АНАЛИТИЧЕСКОЕ РЕШЕНИЕ ГРАНИЧНЫХ ЗАДАЧ
}

\begin{abstract}
Построено кинетическое уравнение, описывающее поведение квантовых ферми-газов с частотой столкновений молекул, пропорциональной молекулярной скорости. Получено аналитическое решение обобщенной задачи Смолуховского, в которой вдали от поверхности заданы градиент температуры и скорость потока массы. Найдены точные формулы для скачков температуры газа, концентрации и химического потенциала. Анализ предельных случаев показывает переход квантового ферми-газа к классическому или вырожденному.
\end{abstract}

Ключевые слова: граничная задача, кинетическое уравнение, разреженный ферми-газ, функция распределения, обобщенная задача Смолуховского, скачки температуры, концентрации и химического потенциала.

\section{1. ВВЕДЕНИЕ}

Задача о скачке температуры на границе раздела разреженного газа и конденсированной среды (твердого тела, жидкости) относится к числу важнейших в кинетической теории газов [1], [2]. Рассмотрение этой задачи восходит к работам Смолуховского, поэтому ее называют задачей Смолуховского. Важность этой проблемы связана прежде всего с многочисленными практическими применениями [2], [3]. Поведение газа вблизи границы раздела представляет и теоретический интерес.

$\mathrm{K}$ задаче Смолуховского тесно примыкает задача об испарении газа (пара) с плоской поверхности. В связи с этим обе задачи в единой постановке естественно называть обобщенной задачей Смолуховского. Для случая классического газа эта задача рассматривалась в работах [1], [3]-[6].

В то же время представляет интерес рассмотрение этой задачи в ситуации, когда следует учесть квантовые эффекты, например при изучении поведения газа Не ${ }^{3}$ вблизи границы раздела газа и конденсированной фазы при низких температурах. Отметим, что оценка величины квантовых эффектов необходима и для выяснения области применимости результатов, полученных в классической постановке.

* Московский педагогический университет, Москва, Россия 
Данная работа посвяшена решению обобшенной задачи Смолуховского для случая ферми-газа. При этом используется подход, аналогичный рассмотренному ранее для задачи об изотермическом скольжении [7].

\section{2. ВЫВОД КИНЕТИЧЕСКОГО УРАВНЕНИЯ}

Рассмотрим широко применяемое кинетическое уравнение в форме БольцманаКрука-Веландера (БКВ) с частотой столкновений молекул, пропорциональной молекулярной скорости [1], [5], [6],

$$
\frac{\partial f}{\partial t}+(\mathbf{v} \nabla) f=\nu_{0} w\left(f_{\mathrm{M}}^{*}-f\right) .
$$

Здесь $f=f(t, \mathbf{r}, \mathbf{v})$ - функция распределения, $\mathbf{v}$ - молекулярная скорость, $w=|\mathbf{v}-\mathbf{u}|$, $\mathbf{u}$ - среднемассовая скорость газа, $\mathbf{u}=n^{-1} \int \mathbf{v} f d \Omega, n$ - концентрация газа, $f_{\mathrm{M}}^{*}$ - максвелловская функция распределения,

$$
f_{\mathrm{M}}^{*}=n_{*}\left(\frac{m}{2 \pi k T_{*}}\right)^{3 / 2} \exp \left[-\frac{m}{2 k T_{*}}\left(\mathbf{v}-\mathbf{u}_{*}\right)^{2}\right]
$$

$\nu_{0}$ - параметр модели, имеюший смысл обратной длины свободного пробега молекул $l, \nu_{0} \sim l^{-1}$. Параметры $n_{*}, T_{*}, \mathbf{u}_{*}$, входяшие в функцию $f_{\mathrm{M}}^{*}$, определяются из требования выполнения законов сохранения числа молекул, импульса и энергии

$$
\begin{aligned}
\int w f d \Omega & =\int w f_{\mathrm{M}}^{*} d \Omega, \\
\int w \mathbf{v} f d \Omega & =\int w \mathbf{v} f_{\mathrm{M}}^{*} d \Omega, \\
\int w \frac{m}{2}(\mathbf{v}-\mathbf{u})^{2} f d \Omega & =\int w \frac{m}{2}(\mathbf{v}-\mathbf{u})^{2} f_{\mathrm{M}}^{*} d \Omega,
\end{aligned}
$$

где $d \Omega=d \Omega_{\mathrm{M}}=d^{3} v$

Отметим, что уравнение (2.1) соответствует предположению о постоянстве (при отсутствии зависимости от скорости) длины свободного пробега молекул, что отвечает модели, в которой молекулы являются твердыми сферами.

Рассмотрим обобщение уравнения (2.1) на случай квантовых ферми-газов. Будем предполагать, что общий вид этого уравнения сохраняется, однако под функцией $f_{\mathrm{M}}^{*}$ необходимо понимать распределение Ферми [8]

$$
f_{\mathrm{F}}^{*}=\left[\exp \left(\frac{m}{2 k T_{*}}\left(\mathbf{v}-\mathbf{u}_{*}\right)^{2}-\frac{\mu_{*}}{k T_{*}}\right)+1\right]^{-1} .
$$

Здесь параметры $\mu_{*}, T_{*}, \mathbf{u}_{*}$ определяются, как и ранее, условиями (2.2). При этом $d \Omega=d \Omega_{\mathrm{F}}=(2 s+1)(2 \pi \hbar)^{-3} d^{3} \mathbf{p}, \quad \hbar-$ постоянная Планка, $s-$ спин частицы, $\mathbf{p}-\mathrm{ee}$ импульс. Будем считать, что массовая скорость газа много меньше средней тепловой 
скорости молекул, а возникаюшие характерные перепады температуры на длине свободного пробега молекул малы по сравнению с температурой газа. В этих предположениях возможна линеаризация задачи. Будем искать функцию распределения в виде $f=f_{\mathrm{F}}^{\mathrm{s}}+\varphi(t, \mathbf{r}, \mathbf{v}) g$, где

$$
f_{\mathrm{F}}^{\mathrm{s}}=\left[\exp \left(\frac{m v^{2}}{2 k T_{\mathrm{s}}}-\frac{\mu_{\mathrm{s}}}{k T_{\mathrm{S}}}\right)+1\right]^{-1}
$$

$\varphi$ - неизвестная функция, $T_{\mathrm{s}}-$ температура поверхности, $\mu_{\mathrm{s}}-$ химический потенциал газа, находящегося в термодинамическом равновесии с конденсированной фазой при температуре $T_{\mathrm{s}}$. Обозначим также

$$
\begin{aligned}
\varepsilon_{\mathrm{S}} & =\frac{m v^{2}}{2 k T_{\mathrm{s}}}-\frac{\mu_{\mathrm{S}}}{k T_{\mathrm{S}}}, \quad \mathbf{c}=\sqrt{\frac{m}{2 k T_{\mathrm{S}}}} \mathbf{v}, \\
\alpha=\frac{\mu_{\mathrm{S}}}{k T_{\mathrm{S}}}, \quad \varepsilon_{*} & =\frac{m}{2 k T_{*}}\left(\mathbf{v}-\mathbf{u}_{*}\right)^{2}-\frac{\mu_{*}}{k T_{*}}, \quad-\infty<\alpha<+\infty .
\end{aligned}
$$

С учетом этих обозначений можно записать

$$
f_{\mathrm{F}}^{*}=\frac{1}{e^{\varepsilon_{*}}+1}, \quad f_{\mathrm{F}}^{\mathrm{s}}=\frac{1}{e^{c^{2}-\alpha}+1}
$$

Линеаризуем функцию $f_{\mathrm{F}}^{*}$, переходя к безразмерным величинам. Заметим, что

$$
\varepsilon_{*}=\frac{T_{\mathrm{S}}}{T_{*}}\left[\frac{m}{2 k T_{\mathrm{S}}}\left(\mathbf{v}-\mathbf{u}_{*}\right)^{2}-\frac{\mu_{*}}{k T_{\mathrm{S}}}\right]=\frac{T_{\mathrm{S}}}{T_{*}}\left[\left(\mathbf{c}-\tilde{\mathbf{u}}_{*}\right)^{2}-\alpha_{*}\right],
$$

где $\tilde{\mathbf{u}}_{*}=\sqrt{m /\left(2 k T_{\mathrm{S}}\right)} \mathbf{u}_{*}, \quad \alpha_{*}=\mu_{*} /\left(k T_{\mathrm{S}}\right)$. В случае вырожденного ферми-газа, когда $\mu>0$, более естественным будет введение безразмерной скорости $\tilde{\mathbf{u}}_{*}=\sqrt{m /(2 \mu)} \mathbf{u}_{*}$, где величина $v_{\mathrm{F}}=\sqrt{2 \mu / m}$ имеет смысл скорости Ферми.

Учитывая, что $T_{*}=T_{\mathrm{S}}+\delta T_{\mathrm{S}}, \alpha_{*}=\alpha+\delta \alpha_{*}$, получаем

$$
\varepsilon_{*}=c^{2}-\alpha-\frac{\delta T_{*}}{T_{\mathrm{S}}}\left(c^{2}-\alpha\right)-\delta \alpha_{*}-2 \mathbf{c} \tilde{\mathbf{u}}_{*},
$$

откуда следует, что

$$
\delta \varepsilon_{*}=-\delta \alpha_{*}-2 \mathbf{c} \tilde{\mathbf{u}}_{*}-\left(c^{2}-\alpha\right) \frac{\delta T_{*}}{T_{\mathrm{S}}},
$$

где $\delta \varepsilon_{*}=\varepsilon_{*}-\varepsilon_{\mathrm{S}}, \varepsilon_{\mathrm{S}}=c^{2}-\alpha$. Следовательно,

$$
f_{\mathrm{F}}^{*}=f_{\mathrm{F}}^{\mathrm{s}}+\left(\frac{\partial f_{\mathrm{F}}^{*}}{\partial \varepsilon_{*}}\right)_{\varepsilon_{*}=\varepsilon_{\mathrm{S}}} \delta \varepsilon_{*}
$$

или

$$
f_{\mathrm{F}}^{*}=f_{\mathrm{F}}^{\mathrm{s}}+g(c ; \alpha)\left[\delta \alpha_{*}+2 \mathbf{c} \tilde{\mathbf{u}}_{*}+\left(c^{2}-\alpha\right) \frac{\delta T_{*}}{T_{\mathrm{S}}}\right],
$$


где

$$
g=-\frac{\partial}{\partial \varepsilon_{\mathrm{S}}} f_{\mathrm{F}}^{\mathrm{S}}=\frac{e^{c^{2}-\alpha}}{\left(e^{c^{2}-\alpha}+1\right)^{2}} .
$$

Поскольку массовая скорость $\mathbf{u}$ много меньше молекулярной скорости $\mathbf{v}$, величину $w$ в линейном приближении [6] можно заменить на $|\mathbf{v}|$.

Введем безразмерные величины $t^{*}=t \nu_{0} \sqrt{2 k T_{\mathrm{s}} / m}$ и $\mathbf{r}^{*}=\mathbf{r} \nu_{0}$. В дальнейшем звездочки у этих величин будем опускать.

Уравнение (2.1) в безразмерных переменных принимает вид

$$
\frac{\partial \varphi}{\partial t}+(\mathbf{c} \nabla) \varphi=c \delta \alpha_{*}+2 c\left(\mathbf{c} \tilde{\mathbf{u}}_{*}\right)+c\left(c^{2}-\alpha\right) \frac{\delta T_{*}}{T_{\mathrm{S}}}-c \varphi .
$$

Параметры этого уравнения находятся из законов сохранения $(2.2)$, которые теперь принимают вид следуюшей системы уравнений:

где

$$
\int(L \varphi) g d^{3} c=0, \quad \int(L \varphi) c^{2} g d^{3} c=0, \quad \int(L \varphi) \mathbf{c} g d^{3} c=0,
$$

$$
L \varphi=c \delta \alpha_{*}+2 \mathbf{c} \tilde{\mathbf{u}}_{*}+c\left(c^{2}-\alpha\right) \frac{\delta T_{*}}{T_{\mathrm{S}}}-c \varphi .
$$

Из этой системы находим

$$
\begin{aligned}
\tilde{\mathbf{u}}_{*} & =\frac{3}{4 \pi r_{1}(\alpha)} \int \varphi \mathbf{c} g c d^{3} c \\
\frac{\delta T_{*}}{T_{\mathrm{s}}} & =-\frac{r_{1}(\alpha)}{2 \pi \Delta(\alpha)} \int \varphi g c d^{3} c+\frac{l(\alpha)}{2 \pi \Delta(\alpha)} \int \varphi g c^{3} d^{3} c, \\
\delta \alpha_{*}-\alpha \frac{\delta T_{*}}{T_{\mathrm{s}}} & =\frac{r_{3}(\alpha)}{2 \pi \Delta(\alpha)} \int \varphi g c d^{3} c-\frac{r_{1}(\alpha)}{2 \pi \Delta(\alpha)} \int \varphi g c^{3} d^{3} c .
\end{aligned}
$$

Здесь

$$
\begin{array}{ll}
r_{1}(\alpha)=4 \int_{0}^{\infty} c \ln \left(1+e^{\alpha-c^{2}}\right) d c, & l(\alpha)=\ln \left(1+e^{\alpha}\right), \\
r_{3}(\alpha)=12 \int_{0}^{\infty} c^{3} \ln \left(1+e^{\alpha-c^{2}}\right) d c, & \Delta(\alpha)=l(\alpha) r_{3}(\alpha)-r_{1}^{2}(\alpha) .
\end{array}
$$

Представим уравнение (2.3) в стандартной форме

$$
\frac{\partial \varphi}{\partial t}+(\mathbf{c} \nabla) \varphi+c \varphi(t, \mathbf{r}, \mathbf{c})=\frac{c}{2 \pi} \int k\left(\mathbf{c}, \mathbf{c}^{\prime}\right) \varphi\left(t, \mathbf{r}, \mathbf{c}^{\prime}\right) d \Omega(\alpha)
$$

где

$$
k=1+3 \frac{l(\alpha)}{r_{1}(\alpha)} \mathbf{c c}^{\prime}+\frac{l^{2}(\alpha)}{\Delta(\alpha)}\left(c^{2}-\frac{r_{1}(\alpha)}{l(a)}\right)\left(c^{\prime 2}-\frac{r_{1}(\alpha)}{l(\alpha)}\right), \quad d \Omega(\alpha)=\frac{g\left(c^{\prime} ; \alpha\right) c^{\prime}}{l(\alpha)} d^{3} c^{\prime} .
$$

ЗАмечАниЕ. При $\alpha \rightarrow-\infty$ уравнение (2.4) переходит в классическое уравнение для одноатомного газа с частотой столкновений молекул, пропорциональной молекулярной скорости [1], [5], которое имеет тот же вид (2.4), но в котором

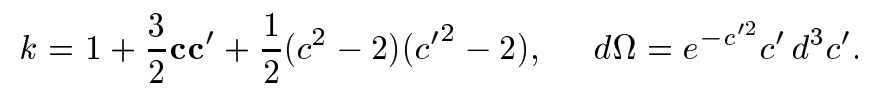

Для доказательства достаточно заметить, что

$$
\lim _{\alpha \rightarrow-\infty} \frac{g(c ; \alpha)}{l(\alpha)}=e^{-c^{2}}, \quad \lim _{\alpha \rightarrow-\infty} \frac{r_{1}(\alpha)}{l(\alpha)}=2, \quad \lim _{\alpha \rightarrow-\infty} \frac{l^{2}(\alpha)}{\Delta(\alpha)}=\frac{1}{2} .
$$




\section{3. ПОСТАНОВКА ЗАДАЧИ}

В рассматриваемой задаче газ занимает полупространство $x>0$ над плоской поверхностью, с которой происходит испарение (конденсация) молекул газа (пара), а также происходит теплообмен между конденсированной фазой и газом. Предположим, что вдали от поверхности сушествуют отличные от нуля градиент температуры, перпендикулярный поверхности (и соответствуюший поток тепла), и некоторая среднемассовая скорость газа, направленная от поверхности или к поверхности (это соответствует испарению или конденсации газа), т.е.

$$
\begin{array}{llrl}
T(x) & =T_{0}+\left(\frac{d T}{d x}\right)_{\infty} x, & & x \rightarrow+\infty, \\
\mathbf{u}(x) & =\left\{u_{\infty}, 0,0\right\}, & & x \rightarrow+\infty .
\end{array}
$$

Температурное поле можно представить с помощью формулы, содержащей величину относительного скачка температуры $\varepsilon_{t}=\left(T_{0}-T_{\mathrm{s}}\right) / T_{\mathrm{S}}$,

$$
T(x)=T_{\mathrm{s}}\left(1+\varepsilon_{t}+k_{t} x\right), \quad x \rightarrow+\infty,
$$

где

$$
k_{t}=\frac{1}{T_{\mathrm{s}}}\left(\frac{d T}{d x}\right)_{\infty}
$$

- относительный градиент температуры.

Обобшенная задача Смолуховского состоит в нахождении величины $\varepsilon_{t}$ как функции $k_{t}$ и $u_{\infty}$. Учитывая линейный характер задачи, можно записать $\varepsilon_{t}=T_{t} k_{t}+T_{u} U, U=$ $\sqrt{m /\left(2 k T_{\mathrm{s}}\right)} u_{\infty}$. Безразмерные величины $T_{t}, T_{u}$ называются коэффициентами скачка температуры. У введенных величин индекс $t$ соответствует температуре, а индекс $u$ скорости испарения (конденсации).

Принимая во внимание стационарный характер задачи и то обстоятельство, что функция $\varphi$ не зависит от координат $y$ и $z$, упростим уравнение (2.4),

$$
\mu \frac{\partial \varphi}{\partial x}+\varphi(x, \mu, c)=\int_{-1}^{1} \int_{0}^{\infty} k\left(\mu, c ; \mu^{\prime}, c^{\prime}\right) \varphi\left(x, \mu^{\prime}, c^{\prime}\right) d \Omega(\alpha)
$$

где

$$
\begin{gathered}
\mu=\frac{c_{x}}{c}, \quad d \Omega(\alpha)=\frac{g\left(c^{\prime} ; \alpha\right)}{l(\alpha)} d \mu^{\prime} d c^{\prime}, \\
k=1+3 \frac{l(\alpha)}{r_{1}(\alpha)} \mu c \mu^{\prime} c^{\prime}+\frac{l^{2}(\alpha)}{\Delta(\alpha)}\left(c^{2}-\frac{r_{1}(\alpha)}{\Delta(\alpha)}\right)\left(c^{\prime 2}-\frac{r_{1}(\alpha)}{\Delta(\alpha)}\right) .
\end{gathered}
$$

Заметим, что уравнение (3.1) в качестве первых трех частных решений имеет инварианты столкновений $\varphi_{1}=1, \varphi_{2}=c^{2}, \varphi_{3}=\mu c$. Кроме того, уравнение (3.1) имеет частное решение, описываюшее перенос тепла в неоднородно нагретом газе,

$$
\varphi_{4}=x\left(c^{2}-\frac{r_{2}(\alpha)}{r_{0}^{2}(\alpha)}\right)-\mu\left[c^{2}-\frac{r_{2}(\alpha)+c \Delta_{1}(\alpha)}{r_{0}^{2}(\alpha)}\right] .
$$


Следовательно, функцией распределения Чепмена-Энскога [1] является функция

$\varphi_{\mathrm{as}}(x, \mu, c)=\varepsilon_{1}+\varepsilon_{2}\left(c^{2}-\frac{3 r_{2}(\alpha)}{5 r_{0}(\alpha)}\right)+\left(u_{1}-k_{t} \frac{\Delta_{1}(\alpha)}{r_{0}^{2}(\alpha)}\right) \mu c+k_{t}(x-\mu)\left(c^{2}-\frac{r_{2}(\alpha)}{r_{0}^{2}(\alpha)}\right)$.

Здесь

$$
\begin{gathered}
\Delta_{1}(\alpha)=r_{2}(\alpha) l(\alpha)-r_{1}(\alpha) r_{0}(\alpha), \quad r_{0}(\alpha)=\frac{3}{2} \int_{0}^{\infty} \ln \left(1+e^{\alpha-c^{2}}\right) d c \\
r_{2}(\alpha)=\frac{15}{2} \int_{0}^{\infty} c^{2} \ln \left(1+e^{\alpha-c^{2}}\right) d c
\end{gathered}
$$

$\varepsilon_{1}, \varepsilon_{2}$ и $u_{1}$ - произвольные постоянные. Выясним физический смысл этих величин.

Линеаризуем локально-равновесную функцию распределения относительно функции $f_{\mathrm{F}}^{\mathrm{S}}$ :

$$
f_{\mathrm{F}}=f_{\mathrm{F}}^{\mathrm{s}}+g\left(\delta \alpha+2 \mathbf{c u}+\left(c^{2}-\alpha\right) \frac{\delta T}{T_{\mathrm{S}}}\right) .
$$

Отметим, что под локально-равновесной понимается фермиевская функция распределения, которая описывает состояние газа с теми же величинами концентрации, кинетической энергии молекулярного движения и среднемассовой скорости газа, что и реальная неравновесная функция распределения. Тогда для определения величин абсолютного скачка химического потенциала $\delta \alpha$ и относительного скачка температуры $\varepsilon_{t}=$ $\delta T(0) / T_{\mathrm{s}}$ получаем с учетом очевидной симметрии следуюшую систему уравнений:

$$
\begin{aligned}
\int\left(\delta \alpha+\left(c^{2}-\alpha\right) \varepsilon_{t}\right) d^{3} c & =\int \varphi g d^{3} c \\
\int\left(\delta \alpha+\left(c^{2}-\alpha\right) \varepsilon_{t}\right) c^{2} d^{3} c & =\int \varphi g c^{2} d^{3} c .
\end{aligned}
$$

Среднемассовая скорость (в направлении оси $x$ ) определяется из уравнения

$$
2 \int \mathbf{c}(\mathbf{c u}) g d^{3} c=\int \mathbf{c} \varphi g d^{3} c .
$$

Из системы уравнений (3.2) находим

$$
\begin{aligned}
\delta \alpha & =\frac{r_{2}(\alpha)-\alpha r_{0}(\alpha)}{\Delta_{2}(\alpha)} \int_{-1}^{1} \int_{0}^{\infty} \varphi g c^{2} d \mu d c-\frac{r_{0}(\alpha)-\alpha s(\alpha)}{\Delta_{2}(\alpha)} \int_{-1}^{1} \int_{0}^{\infty} \varphi g c^{4} d \mu d c, \\
\varepsilon_{t} & =-\frac{r_{0}(\alpha)}{\Delta_{2}(\alpha)} \int_{-1}^{1} \int_{0}^{\infty} \varphi g c^{2} d \mu d c+\frac{s(\alpha)}{\Delta_{2}(\alpha)} \int_{-1}^{1} \int_{0}^{\infty} \varphi g c^{4} d \mu d c,
\end{aligned}
$$

где

$$
s(\alpha)=\int_{0}^{\infty} \frac{d c}{e^{c^{2}-\alpha}+1}, \quad \Delta_{2}(\alpha)=s(\alpha) r_{2}(\alpha)-r_{0}^{2}(\alpha) .
$$

Подставляя в эти равенства $\varphi=\varphi_{\mathrm{as}}$, получаем

$$
\varepsilon_{2}=\varepsilon_{t}, \quad \varepsilon_{1}=\delta \alpha-\left(\alpha-\frac{3 r_{2}(\alpha)}{5 r_{0}(\alpha)}\right) \varepsilon_{t}
$$


Из уравнения (3.3) находим среднемассовую скорость

$$
\mathbf{u}=\frac{3}{4 \pi r_{0}(\alpha)} \int \mathbf{c} \varphi g d^{3} c
$$

откуда для интересуюшей нас $x$-компоненты скорости будем иметь

$$
u_{x}=\frac{3}{2 r_{0}(\alpha)} \int_{-1}^{1} \int_{0}^{\infty} \varphi g \mu c^{3} d \mu d c
$$

Подставляя в последнее равенство $\varphi=\varphi_{\text {as }}$, находим $u_{1}=2 U$. Таким образом, распределение Чепмена-Энскога построено.

Помимо скачка температуры в обобщенной задаче Смолуховского важнейшей характеристикой является величина относительного скачка концентрации $\varepsilon_{n}$ (индекс $n$ соответствует концентрации),

$$
\varepsilon_{n}=\frac{n_{0}-n_{\mathrm{S}}}{n_{\mathrm{S}}}=\int \varphi g d^{3} c\left(\int f_{\mathrm{F}}^{(0)} d^{3} c\right)^{-1}=\frac{3}{2 r_{0}(\alpha)} \int_{-1}^{1} \int_{0}^{\infty} \varphi g c^{2} d^{3} c
$$

или

$$
\varepsilon_{n}=\frac{3 s(\alpha)}{2 r_{0}(\alpha)}\left[\varepsilon_{1}+\left(\frac{r_{0}(\alpha)}{s(\alpha)}-\frac{3 r_{2}(\alpha)}{5 r_{0}(\alpha)}\right) \varepsilon_{t}\right]
$$

Теперь, предполагая отражение молекул от стенки чисто диффузным, запишем граничные условия в обобщенной задаче Смолуховского,

$$
\begin{gathered}
\varphi(0, \mu, c)=0, \quad 0<\mu<1, \\
\varphi(x, \mu, c)=\varphi_{\text {as }}(x, \mu, c)+o(1), \quad x \rightarrow+\infty, \quad-1<\mu<0 .
\end{gathered}
$$

\section{4. СВЕДЕНИЕ К ОДНОСКОРОСТНОЙ ЗАДАЧЕ. РАЗДЕЛЕНИЕ} ПЕРЕМЕННЫХ. ХАРАКТЕРИСТИЧЕСКОЕ УРАВНЕНИЕ

Будем искать решение задачи (3.1), (3.4) в виде

$$
\varphi=h_{1}(x, \mu)+c h_{2}(x, \mu)+\left(c^{2}-\frac{r_{1}(\alpha)}{l(\alpha)}\right) h_{3}(x, \mu) .
$$

Полученную задачу представим в векторном виде,

$$
\begin{gathered}
\mu \frac{\partial h}{\partial x}+h(x, \mu)=\frac{1}{2} \int_{-1}^{1} K\left(\mu, \mu^{\prime} ; \alpha\right) h\left(x, \mu^{\prime}\right) d \mu^{\prime}, \\
h(0, \mu)=\mathbf{0}, \quad 0<\mu<1, \\
h(x, \mu)=h_{\mathrm{as}}(x, \mu)+o(1), \quad x \rightarrow+\infty, \quad-1<\mu<0 .
\end{gathered}
$$

Здесь матрищы-строки $\mathbf{0}=[000]^{\mathrm{t}}, h=\left[h_{1} h_{2} h_{3}\right]^{\mathrm{t}}$, матрища-столбец

$$
h_{\mathrm{as}}=\left[\begin{array}{c}
\varepsilon_{1}+\varepsilon_{t}\left(\frac{r_{1}(\alpha)}{l(\alpha)}-\frac{3 r_{2}(\alpha)}{5 r_{0}(\alpha)}\right)+k_{t}(x-\mu)\left(\frac{r_{1}(\alpha)}{l(\alpha)}-\frac{r_{2}(\alpha)}{r_{0}(\alpha)}\right) \\
\left(2 U-\frac{\Delta_{1}(\alpha)}{r_{0}^{2}(\alpha)} k_{t}\right) \mu \\
\varepsilon_{t}+k_{t}(x-\mu)
\end{array}\right],
$$


матрица

$$
K\left(\mu, \mu^{\prime} ; \alpha\right)=\left[\begin{array}{ccc}
1 & \frac{r_{0}(\alpha)}{l(\alpha)} & 0 \\
3 \mu \mu^{\prime} \frac{r_{0}(\alpha)}{r_{1}(\alpha)} & 3 \mu \mu^{\prime} & 3 \mu \mu^{\prime} \frac{\Delta_{1}(\alpha)}{l(\alpha) r_{1}(\alpha)} \\
0 & \frac{\Delta_{1}(\alpha)}{\Delta(\alpha)} & 1
\end{array}\right]
$$

Перепишем уравнение (4.1) в виде

$$
\mu \frac{\partial h}{\partial x}+h(x, \mu)=\frac{1}{2} K_{0} n_{0}[h]+\frac{1}{2} \mu K_{1} n_{1}[h],
$$

где

$$
\begin{gathered}
n_{j}[h]=\int_{-1}^{1} \mu^{j} h(x, \mu) d \mu, \quad j=0,1, \\
K_{0}=\left[\begin{array}{ccc}
1 & \frac{r_{0}(\alpha)}{l(\alpha)} & 0 \\
0 & 0 & 0 \\
0 & \frac{\Delta_{1}(\alpha)}{\Delta(\alpha)} & 1
\end{array}\right], \quad K_{1}=3\left[\begin{array}{cccc}
0 & 0 & 0 \\
\frac{r_{0}(\alpha)}{r_{1}(\alpha)} & 1 & \frac{\Delta_{1}(\alpha)}{l(\alpha) r_{1}(\alpha)} \\
0 & 0 & 0
\end{array}\right] .
\end{gathered}
$$

Обший метод разделения переменных приводит к выражению

$$
h_{\eta}=e^{-x / \eta} \Phi(\eta, \mu)
$$

подставляя которое в (4.2), получим характеристическое уравнение

$$
(\eta-\mu) \Phi(\eta, \mu)=\frac{1}{2} \eta K_{0} n_{0}[\Phi]+\frac{1}{2} \mu \eta K_{1} n_{1}[\Phi] .
$$

Интегрируя по $\mu$ последнее уравнение, находим

$$
n_{1}[\Phi]=\eta\left(E-K_{0}\right) n_{0}[\Phi]
$$

где $E$ - единичная матрица. Следовательно, характеристическое уравнение упрошается и принимает вид

$$
(\eta-\mu) \Phi(\eta, \mu)=\frac{1}{2} \eta D(\eta \mu) n_{0}[\Phi]
$$

где

$$
\begin{gathered}
D(\mu \eta)=K_{0}+d(\alpha) \mu \eta E_{1}, \quad E_{1}=\left[\begin{array}{lll}
0 & 0 & 0 \\
0 & 1 & 0 \\
0 & 0 & 0
\end{array}\right] \\
d(\alpha)=3\left[1-\frac{r_{0}^{2}(\alpha) \Delta(\alpha)+\Delta_{1}(\alpha)}{l(\alpha) r_{1}(\alpha) \Delta(\alpha)}\right] .
\end{gathered}
$$

При $\eta \in(-1,1)$ из уравнения $(4.3)$ в пространстве обобщенных функций [9] находим собственные векторы [10] непрерывного спектра $\Phi(\eta, \mu)=F(\eta, \mu) n_{0}[\Phi]$, где

$$
F(\eta, \mu)=\frac{1}{2} \eta D(\mu \eta) P \frac{1}{\eta-\mu}+\Lambda(\eta) \delta(\eta-\mu)
$$


- собственная матрица, обобшенная функция $P \frac{1}{x}$ - главное значение интеграла от $1 / x$, $\delta(x)-\delta$-функция Дирака, $\Lambda(z)$ - дисперсионная матрица,

$$
\Lambda(z)=E+z \frac{1}{2} \int_{-1}^{1} \frac{D(\mu z)}{\mu-z} d \mu .
$$

Представим дисперсионную матрицу в явном виде $\Lambda(z)=\lambda_{\mathrm{c}}(z) D\left(z^{2}\right)+K_{2}$, где

$$
K_{2}=\left[\begin{array}{ccc}
0 & -\frac{r_{0}(\alpha)}{l(\alpha)} & 0 \\
0 & 1 & 0 \\
0 & -\frac{\Delta_{1}(\alpha)}{\Delta(\alpha)} & 0
\end{array}\right], \quad \lambda_{\mathrm{c}}(z)=1+z \frac{1}{2} \int_{-1}^{1} \frac{d u}{u-z} .
$$

Ее определитель (дисперсионная функция) выражается формулой

$$
\lambda(z)=\operatorname{det} \Lambda(z)=\lambda_{c}(z) \omega(z ; \alpha)
$$

где $\omega(z ; \alpha)=1+d(\alpha) z^{2} \lambda_{\mathrm{c}}(z)$.

\section{5. СОБСТВЕННЫЕ ВЕКТОРЫ ДИСКРЕТНОГО СПЕКТРА}

Дискретным спектром по определению (см., например, [10], [11]) называют множество нулей дисперсионной функции. Найдем нули функции $\omega(z ; \alpha)$, лежашие вне разреза $[-1,1]$. Очевидно, что $\omega(\infty ; \alpha)=1-d(\alpha) / 3>0$ при всех действительных $\alpha$. Возьмем контур $\gamma_{\varepsilon}$, охватываюший разрез $[-1,1]$, отстояший от него на расстоянии $\varepsilon$ и такой, что внутри контура нет нулей функции $\omega(z ; \alpha)$. Число $N$ нулей функции $\omega(z ; \alpha)$ вне контура согласно приншипу аргумента [12] равно прирашению аргумента этой функции, т.е. $N=(2 \pi)^{-1} \Delta_{\gamma_{\varepsilon}} \arg \omega(z ; \alpha)$, где символ $\Delta_{\gamma_{\varepsilon}} f$ означает прирашение $f$ вдоль контура $\gamma_{\varepsilon}$. Переходя к пределу при $\varepsilon \rightarrow 0$ в этом равенстве, получаем

$$
N=\frac{1}{2 \pi} \Delta_{(-1,1)} \arg \frac{\omega^{+}(\mu ; \alpha)}{\omega^{-}(\mu ; \alpha)}
$$

где $\omega^{ \pm}(\mu ; \alpha)$ - граничные значения функции $\omega(z ; \alpha)$ сверху и снизу на интервале $(-1,1)$,

$$
\omega^{ \pm}(\mu ; \alpha)=\omega(\mu ; \alpha) \pm i \frac{\pi}{2} d(\alpha) \mu^{3} .
$$

Учитывая, что $\operatorname{Re} \omega^{+}$является четной функцией, a $\operatorname{Im} \omega^{+}-$нечетной, имеем

$$
N=\frac{1}{\pi} \Delta_{(0,1)} \arg G(\mu ; \alpha), \quad G=\frac{\omega^{+}}{\omega^{-}} .
$$

Пусть $\varepsilon(\mu ; \alpha)=\arg \omega^{+}(\mu ; \alpha)$ - главное значение аргумента, фиксированное условием $\varepsilon(0 ; \alpha)=0$. Так как $\bar{\omega}^{+}=\omega^{-},\left|\omega^{+}\right|=\left|\omega^{-}\right|$, то $\arg G(\mu ; \alpha)=2 \varepsilon(\mu ; \alpha)$, поэтому $N=$ $2 \pi^{-1} \Delta_{(0,1)} \varepsilon(\mu ; \alpha)$. Можно показать, что угол $\varepsilon(\mu ; \alpha)$ на отрезке $[0,1]$ имеет прирашение, равное $\pi$, независимо от величины параметра $\alpha$, следовательно, число нулей $N$ равно 
двум. Обозначим эти нули через $\pm \eta_{0}\left(\eta_{0}>1\right)$. В силу равенства $\omega(z ; \alpha)=\omega(\bar{z} ; \alpha)$ нули действительны. Согласно (4.3) этим нулям отвечают два собственных решения

$$
h_{\eta_{0}}(x, \mu)=\frac{1}{2}\left( \pm \eta_{0}\right) \frac{D\left( \pm \eta_{0} \mu\right)}{ \pm \eta_{0}-\mu} e^{-x /\left( \pm \eta_{0}\right)} n\left( \pm \eta_{0}\right), \quad n(\eta)=n_{0}[\Phi] .
$$

Далее понадобится убьвающее решение $h_{\eta_{0}}$. Подставляя $h_{\eta_{0}}$ в уравнение (4.1), получаем однородное уравнение

$$
\Lambda\left(\eta_{0}\right) n\left(\eta_{0}\right)=\mathbf{0}
$$

определитель которого равен нулю: $\lambda\left(\eta_{0}\right)=\operatorname{det} \Lambda\left(\eta_{0}\right)=0$. Из уравнения (5.1) находим

$$
n\left(\eta_{0}\right)=\left[\begin{array}{c}
-r_{0}(\alpha) \eta_{0} t\left(\eta_{0}\right) l^{-1}(\alpha) \\
\lambda_{c}\left(\eta_{0}\right) \\
-\eta_{0} t\left(\eta_{0}\right) \Delta_{1}(\alpha) \Delta^{-1}(\alpha)
\end{array}\right], \quad t(z)=\frac{1}{2} \int_{-1}^{1} \frac{d u}{u-z}
$$

Заметим, что

$$
D\left(\mu \eta_{0}\right) n\left(\eta_{0}\right)=\left[\begin{array}{c}
r_{0}(\alpha) l^{-1}(\alpha) \\
-\mu \eta_{0}^{-1} \\
\Delta_{1}(\alpha) \Delta^{-1}(\alpha)
\end{array}\right]
$$

Следовательно, частное решение $h_{\eta_{0}}$ построено в явном виде,

$$
h_{\eta_{0}}(x, \mu)=\frac{\eta_{0}}{2\left(\eta_{0}-\mu\right)} e^{-x / \eta_{0}}\left[\begin{array}{c}
r_{0}(\alpha) l^{-1}(\alpha) \\
-\mu \eta_{0}^{-1} \\
\Delta_{1}(\alpha) \Delta^{-1}(\alpha)
\end{array}\right] .
$$

В книге [10] показано, что $\lambda_{\mathrm{c}}(z)$ имеет лиш двойной нуль в точке $z=\infty$. Согласно (4.3) эта точка является 4-кратной точкой дискретного спектра. Ей отвечают следуюшие частные решения:

$$
\begin{gathered}
h^{(1)}(x, \mu)=\left[\begin{array}{l}
1 \\
0 \\
0
\end{array}\right], \quad h^{(2)}(x, \mu)=\mu\left[\begin{array}{l}
0 \\
1 \\
0
\end{array}\right], \\
h^{(3)}(x, \mu)=\left[\begin{array}{l}
0 \\
0 \\
1
\end{array}\right], \quad h^{(4)}(x, \mu)=(x-\mu)\left[\begin{array}{c}
-\Delta_{1}(\alpha)\left(l(\alpha) r_{0}(\alpha)\right)^{-1} \\
0 \\
1
\end{array}\right] .
\end{gathered}
$$

Эти частные решения соответствуют четырем частным решениям $\varphi_{i}, i=1,2,3,4$, кинетического уравнения, физический смысл которых был выяснен выше. 


\section{6. РАЗЛОЖЕНИЕ РЕШЕНИЯ ГРАНИЧНОЙ ЗАДАЧИ ПО СОБСТВЕННЫМ ВЕКТОРАМ. КРАЕВАЯ ЗАДАЧА РИМАНА-ГИЛЬБЕРТА}

Покажем, что решение граничной задачи (4.1) записывается в виде разложения

$$
h(x, \mu)=h_{\mathrm{as}}(x, \mu)+A_{0} h_{\eta_{0}}(x, \mu)+\int_{0}^{\infty} e^{-x / \eta} F(\eta, \mu) A(\eta) d \eta,
$$

где неизвестными являются постоянные $A_{0}, \varepsilon_{1}, \varepsilon_{t}$ и вектор-функция $A(\eta)$ с элементами $A_{j}(\eta), j=1,2,3$.

Разложение (6.1) можно представить в виде

$$
\begin{aligned}
h(x, \mu)= & h_{\mathrm{as}}(x, \mu)+A_{0} h_{\eta_{0}}(x, \mu)+e^{-x / \mu} \Lambda(\mu) A(\mu) \theta_{+}(\mu)+ \\
& +\frac{1}{2} \int_{0}^{1} e^{-x / \eta} \eta D(\mu \eta) A(\eta) \frac{d \eta}{\eta-\mu}
\end{aligned}
$$

где $\theta_{+}(\mu)=1, \mu \in(0,1) ; \theta_{+}(\mu)=0, \mu \notin(-1,0)$. Подставляя разложение (6.2) в первое из граничных условий (см. (4.1)), приходим к сингулярному интегральному уравнению с ядром Коши [12],

$$
h_{\mathrm{as}}(0, \mu)+A_{0} h_{\eta_{0}}(0, \mu)+\Lambda(\mu) A(\mu)+\frac{1}{2} \int_{0}^{1} \eta D(\mu \eta) A(\eta) \frac{d \eta}{\eta-\mu}=\mathbf{0}, \quad 0<\mu<1 .
$$

Введем вспомогательную вектор-функцию

$$
N(z)=\frac{1}{2} \int_{0}^{1} \eta D(z \eta) A(\eta) \frac{d \eta}{\eta-z}
$$

и матрицу $P(z)=\Lambda(z) D^{-1}\left(z^{2}\right)$. С помощью граничных значений $N(z), \Lambda(z), P(z)$ и соответствующих формул Сохоцкого сведем уравнение (6.3) к неоднородной векторной краевой задаче Римана-Гильберта

$$
\begin{aligned}
& P^{+}(\mu)\left[N^{+}(\mu)+h_{\mathrm{as}}(0, \mu)+A_{0} h_{\eta_{0}}(0, \mu)\right]= \\
& \quad=P^{-}(\mu)\left[N^{-}(\mu)+h_{\mathrm{as}}(0, \mu)+A_{0} h_{\eta_{0}}(0, \mu)\right], \quad 0<\mu<1 .
\end{aligned}
$$

Сначала решим соответствуюшую однородную краевую задачу

$$
P^{+}(\mu) X^{+}(\mu)=P^{-}(\mu) X^{-}(\mu), \quad 0<\mu<1 .
$$

Метод решения таких задач был развит в работе [5], поэтому решение задачи (6.6) приведем без вывода,

$$
X(z)=\left[\begin{array}{ccc}
U(z) & \frac{r_{0}(\alpha)}{l(\alpha)}[U(z)-V(z)] & 0 \\
0 & V(z) & 0 \\
0 & \frac{\Delta_{1}(\alpha)}{\Delta(\alpha)}[U(z)-V(z)] & U(z)
\end{array}\right],
$$


где

$$
\begin{aligned}
U(z) & =z e^{-u(z)}, & V(z) & =z e^{-v(z)} \\
u(z) & =\frac{1}{\pi} \int_{0}^{1} \frac{\theta(u)-\pi}{u-z} d u, & v(z) & =\frac{1}{\pi} \int_{0}^{1} \frac{\varepsilon(u ; \alpha)-\pi}{u-z} d u,
\end{aligned}
$$

$\theta(u)=\arg \lambda_{\mathrm{c}}^{+}(u), \theta(0)=0, \varepsilon(u ; \alpha)=\arg \omega^{+}(u ; \alpha), \varepsilon(0 ; \alpha)=0$.

Вернемся к решению неоднородной задачи (6.5). Подставляя матрицу $P^{+}(\mu)$, найденную на основании (6.6), в (6.5), приходим к задаче определения аналитической вектор-функции по нулевому скачку. Общее решение этой задачи имеет вид

$$
N(z)=-h_{\mathrm{as}}(0, z)-A_{0} h_{\eta_{0}}(0, z)+X(z)\left[\frac{1}{z-\eta_{0}} B+C\right],
$$

где $B=\left[b_{1} b_{2} b_{3}\right]^{\mathrm{t}}, C=\left[c_{1} c_{2} c_{3}\right]^{\mathrm{t}}-$ векторы с произвольными элементами.

Неизвестная вектор-функция $A(\eta)$ находится из формулы Сохоцкого

$$
N^{+}(\mu)-N^{-}(\mu)=\pi i \mu D\left(\mu^{2}\right) A(\mu), \quad 0<\mu<1
$$

после подстановки решения (6.7) в (6.8).

Решение (6.7) имеет простые полюсы в точке $\eta_{0}$ и в точке $z=\infty$, в то время как вектор-функция $N(z)$, введенная равенством (6.4), в точке $\eta_{0}$ является аналитической, а в точке $z=\infty$ исчезает ее первый (верхний) и третий (нижний) элементы, а второй элемент имеет конечный предел. Чтобы общее решение можно было принять в качестве вспомогательной функции (6.4), устраним указанные особенности общего решения и приравняем пределы у второго элемента в точке $z=\infty$. При этом получим

$$
B=\frac{\eta_{0} A_{0}}{2 V\left(\eta_{0}\right)}\left[\begin{array}{c}
-\frac{r_{0}(\alpha)}{l(\alpha)} \\
1 \\
-\frac{\Delta_{1}(\alpha)}{\Delta(\alpha)}
\end{array}\right], \quad C=\left[\begin{array}{c}
\frac{\Delta_{1}(\alpha)}{l(\alpha) r_{0}(\alpha)} \\
2 U-\frac{\Delta_{1}(\alpha)}{r_{0}^{2}(\alpha)} k_{t} \\
-k_{t}
\end{array}\right]
$$

\section{7. СКАЧКИ ТЕМПЕРАТУРЫ И КОНЦЕНТРАЦИИ. \\ АНАЛИЗ ПРЕДЕЛЬНЫХ СЛУЧАЕВ}

Опуская громоздкие преобразования, приведем формулы для скачка температуры $\varepsilon_{t}$ и для коэффициента $\varepsilon_{1}$,

$$
\varepsilon_{t}(\alpha)=T_{t}(\alpha) k_{t}+T_{u}(\alpha) 2 U, \quad \varepsilon_{1}(\alpha)=M_{t}(\alpha) k_{t}+M_{u}(\alpha) 2 U
$$

где

$$
\begin{array}{crl}
T_{t}(\alpha)=U_{1}-\frac{\Delta_{1}(\alpha)}{r_{0}^{2}(\alpha)} T_{u}(\alpha), & T_{u}(\alpha)=\frac{\Delta_{1}(\alpha)}{\Delta(\alpha)}\left[V_{1}(\alpha)-U_{1}-\eta_{0}\right], \\
M_{t}(\alpha)=-\frac{2 r_{2}(\alpha)}{5 r_{0}(\alpha)} U_{1}-\frac{\Delta_{1}(\alpha)}{r_{0}^{2}(\alpha)} M_{u}(\alpha), & M_{u}(\alpha)=T_{u}(\alpha)\left[\frac{\Delta_{3}(\alpha)}{\Delta_{1}(\alpha)}+\frac{3 r_{2}(\alpha)}{5 r_{0}(\alpha)}\right] \\
\Delta_{3}(\alpha)=r_{0}(\alpha) r_{3}(\alpha)-r_{1}(\alpha) r_{2}(\alpha), & U_{1}=-\frac{1}{\pi} \int_{0}^{1}(\theta(u)-\pi) d u=0.71045 \ldots, \\
V_{1}(\alpha)=-\frac{1}{\pi} \int_{0}^{1}(\varepsilon(u, \alpha)-\pi) d u .
\end{array}
$$


Подставляя $\varepsilon_{1}$ в полученную выше формулу для скачка концентрации $\varepsilon_{n}$, приведем последнюю к виду

$$
\varepsilon_{n}(\alpha)=N_{t}(\alpha) k_{t}+N_{u}(\alpha) 2 U
$$

где

$$
\begin{aligned}
& N_{t}(\alpha)=-\frac{3 \Delta_{0}(\alpha)}{2 r_{0}^{2}(\alpha)} U_{1}-\frac{\Delta_{1}(\alpha)}{r_{0}^{2}(\alpha)} N_{u}(\alpha) \\
& N_{u}(\alpha)=T_{u}(\alpha) \frac{3 s(\alpha)}{2 r_{0}(\alpha)}\left(\frac{\Delta_{3}(\alpha)}{\Delta_{1}(\alpha)}+\frac{r_{0}(\alpha)}{s(\alpha)}\right) \\
& \Delta_{0}(\alpha)=s(\alpha) r_{2}(\alpha)-r_{0}^{2}(\alpha)
\end{aligned}
$$

$\mathrm{C}$ помошью $\varepsilon_{1}$ и $\varepsilon_{t}$ находится и величина скачка химического потенциала

$$
\delta \alpha=\varepsilon_{1}(\alpha)+\left(\alpha-\frac{3 r_{2}(\alpha)}{5 r_{0}(\alpha)}\right) \varepsilon_{t}
$$

Зависимости кинетических коэффициентов $T_{t}, T_{u}, N_{t}, N_{u}$ от величины химического потенциала $\alpha$ представлены на рис. $1-4$.

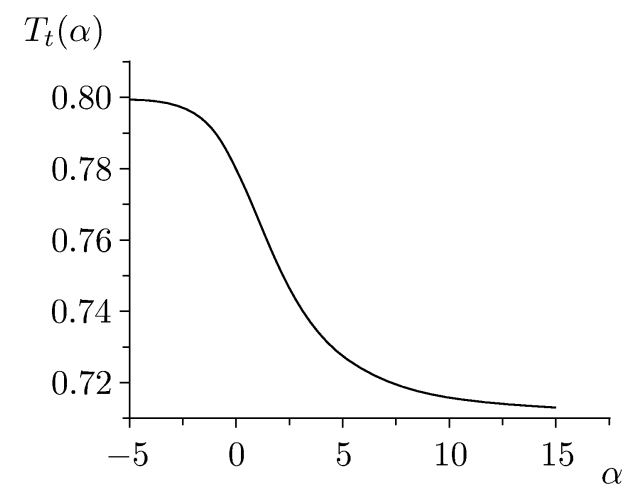

Рис. 1

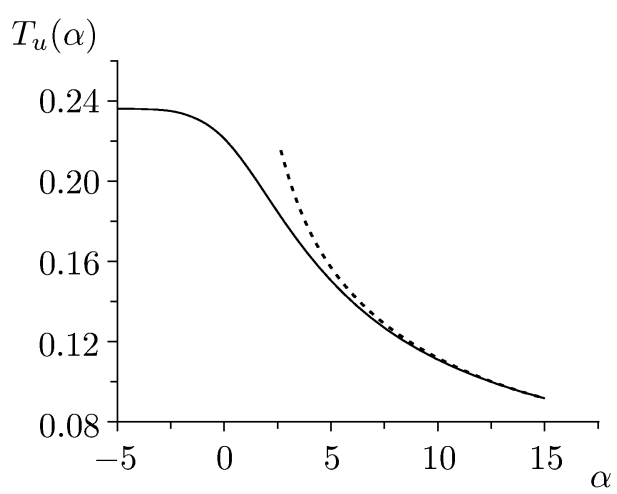

Рис. 2

Рассмотрим предельные случаи кинетических коэффициентов при $\alpha \rightarrow-\infty$ и $\alpha \rightarrow$ $+\infty$. Находя пределы $\varepsilon_{t}(\alpha)$ и $\varepsilon_{n}(\alpha)$ при $\alpha \rightarrow-\infty$, в точности получаем значения кинетических коэффициентов [5] для больцмановского газа.

В случае $\alpha \rightarrow+\infty$ для оценки интегралов, входящих в кинетические коэффициенты, воспользуемся асимптотической формулой (см. [8], с. 191, формула $(58,1))$

$$
\int_{0}^{\infty} \frac{f(x) d x}{e^{x-\alpha}+1}=\int_{0}^{\alpha} f(x) d x+\frac{\pi^{2}}{6} f^{\prime}(\alpha)+\frac{7 \pi^{2}}{360} f^{\prime \prime \prime}(\alpha)+\cdots
$$




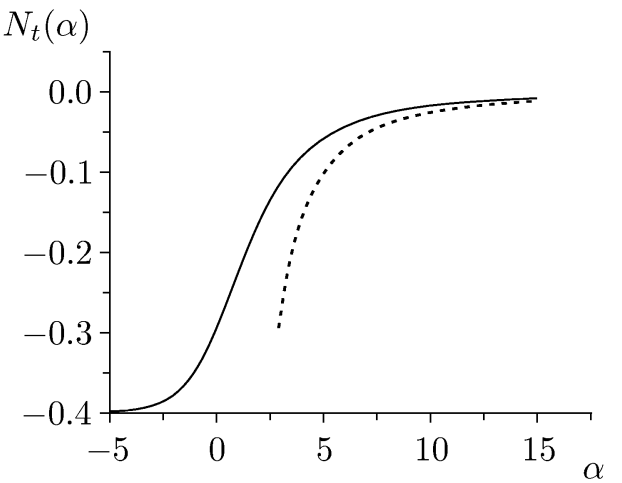

Рис. 3

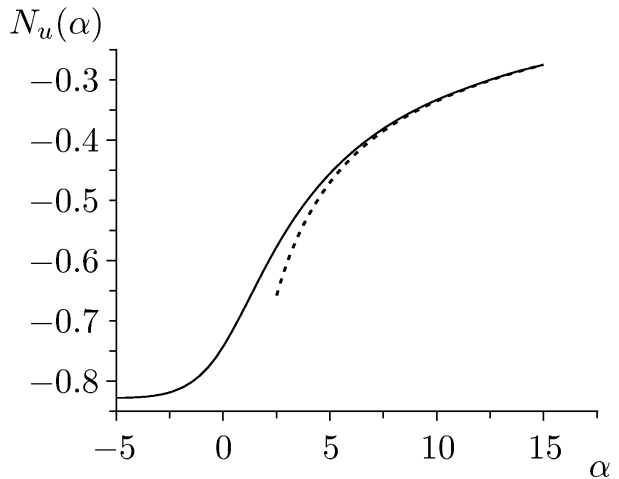

Рис. 4

с помощью которой найдем, что $d(\alpha) \rightarrow 0$ при $\alpha \rightarrow+\infty$; следовательно, $\eta_{0}(\alpha) \rightarrow 1, \alpha \rightarrow$ $+\infty$, причем $\eta_{0}(-\infty)=1+3 \cdot 10^{-40}[1]$. На основании формулы $(7.1)$ найдем, что при $\alpha \rightarrow+\infty$

$$
\begin{aligned}
r_{1}(\alpha) & =\alpha^{2}+\frac{\pi^{2}}{3}+\cdots, & r_{3}(\alpha) & =\alpha^{3}+\pi^{2} \alpha+\cdots, \\
\Delta(\alpha) & =\frac{\pi^{2}}{3}\left(\alpha^{2}-\frac{\pi^{2}}{3}+\cdots\right), & r_{2}(\alpha) & =\alpha^{5 / 2}+\frac{5}{8} \pi^{2} \alpha^{1 / 2}+\cdots, \\
r_{0}(\alpha) & =\alpha^{3 / 2}+\frac{1}{8} \pi^{2} \alpha^{-1 / 2}+\cdots, & s(\alpha) & =\alpha^{1 / 2}-\frac{1}{24} \pi^{2} \alpha^{-1 / 2}+\cdots, \\
\Delta_{1}(\alpha) & =\frac{1}{6} \pi^{2}\left(\alpha^{3 / 2}-\frac{1}{4} \pi^{2} \alpha^{-1 / 2}+\cdots\right), & \Delta_{3}(\alpha) & =\frac{1}{6} \pi^{2}\left(\alpha^{5 / 2}+\frac{1}{2} \pi^{2} \alpha^{1 / 2}+\cdots\right) .
\end{aligned}
$$

С учетом этих равенств находим асимптотику кинетических коэффициентов при $\alpha \rightarrow$ $+\infty$ :

$$
\begin{array}{ll}
T_{t}(\alpha) \approx U_{1}, & T_{u}(\alpha) \approx-\frac{U_{1}}{2} \frac{1}{\sqrt{\alpha}} \\
N_{t}(\alpha) \approx-\frac{3}{8} \pi^{2} U_{1} \frac{1}{\alpha^{2}}, & N_{u}(\alpha) \approx-\frac{3 U_{1}}{2} \frac{1}{\sqrt{\alpha}}
\end{array}
$$

На рис. 2-4 последние три асимптотики изображены пунктиром.

Случай $\alpha \rightarrow+\infty$ соответствует случаю вырожденного ферми-газа. При этом использованное ранее введение безрамерной скорости газа $\tilde{\mathbf{u}}_{*}=\sqrt{m /\left(2 k T_{\mathrm{S}}\right)} \mathbf{u}_{*}$ становится неэффективным, так как энергия молекул определяется в этом случае не температурой газа, а величиной энергии Ферми. Более естественным будет введение безразмерной скорости $\tilde{\mathbf{u}}=\sqrt{m /(2 \alpha)} \mathbf{u}_{*}$, что возможно только при $\alpha>0$, т.е. не во всем диапазоне значений химического потенциала. При этом формулы для коэффициентов $T_{u}(\alpha)$, $N_{u}(\alpha)$ принимают вид $T_{u}^{*}(\alpha)=\sqrt{\alpha} T_{u}(\alpha), N_{u}^{*}(\alpha)=\sqrt{\alpha} N_{u}(\alpha)$. Согласно приведенной выше асимптотике $T_{u}^{*}(\alpha)=-U_{1} / 2, N_{u}^{*}(\alpha)=-3 U_{1} / 2$. 
Из приведенных рисунков видно, что поведение кинетических коэффициентов принципиально различно в трех диапазонах. Например, для коэффициента $T_{t}(\alpha)$ (рис. 1 ) при $\alpha<-4$ наблюдается переход к классическому значению $T_{t}(-\infty)=0.79$, а в случае $\alpha>10$ наблюдается поведение, характерное для вырожденного ферми-газа, при этом $T_{t}(+\infty)=U_{1}=0.71045$. Область значений $-4<\alpha<10$ соответствует переходному режиму от классического к вырожденному ферми-газу.

Благодарности. Работа выполнена при частичной финансовой поддержке РФФИ (грант № 99-01-00336).

\section{Список литературы}

[1] K. Черчиньяни. Теория и приложения уравнения Больцмана. М.: Мир, 1977.

[2] М. Н. Коган. Динамика разреженного газа. Кинетическая теория. М.: Наука, 1967.

[3] О. А. Коленчии. Тепловая аккомодация систем газ - твердое тело. Минск: Наука и техника, 1977.

[4] А. В. Латыишев, А.А. Юиканов. ТМФ. 1993. Т. 95. № 3. С. 530.

[5] А. В. Латыиее, А. А. Юиканов. Изв. РАН. Сер. МЖГ. 1996. Т. 1996. № 3. С. 140.

[6] А.В. Латышев, А. А. Юшканов. ЖЭТФ. 1998. Т. 114. № 3(9). С. 956.

[7] А. В. Латышев, А. А. Юиканов. ТМФ. 2001. Т. 129. № 3. С. 491.

[8] Л. Д. Ландау, Е. М. Лифиич. Статистическая физика. Ч. 1. М.: Наука, 1976.

[9] В. С. Владимиров, В. В. Жаринов. Уравнения математической физики. М.: Наука, 2001.

[10] К. Кейз, П. Цвайфель. Линейная теория переноса. М.: Мир, 1972.

[11] Т. А. Гермогенова. О полноте системы собственных функций характеристического уравнения теории переноса. Препринт № 103. М.: ИПМ им. М. В. Келдыша, 1976.

[12] Ф. Д. Гахов. Краевые задачи. М.: Наука, 1977. 